Bull. Korean Math. Soc. 47 (2010), No. 2, pp. 287-293

DOI 10.4134/BKMS.2010.47.2.287

\title{
CHARACTERIZATIONS ON CHAIN RECURRENCES
}

\author{
Jong-Suh Park And Se-Hyun Ku
}

\begin{abstract}
It is well known that there is a residual subset $J$ of the space of $C^{1}$-diffeomorphisms on a compact Riemannian manifold $M$ such that the maps $f \mapsto$ chain recurrent set of $f$ and $f \mapsto$ number of chain components of $f$ are continuous on $J$. In this paper we get the flow version of the above results on diffeomorphisms.
\end{abstract}

\section{Introduction}

The interesting problems in the study of dynamical systems are to understand the structure of various invariant sets of a given system, and to explain how these sets varies as the given system changes. We naturally focuss on the region of the phase space consisting of the chain recurrent orbits. Roughly speaking, a dynamical system is said to be chain recurrent if every orbit can be made periodic by allowing arbitrary small jumps at arbitrary large times.

Conley $[3,4]$ introduced the notion of chain recurrence in study of flows on compact spaces. Block and Franke [1] considered the chain recurrent set of a continuous map from the interval to itself. Bronstein and Kopanskii [2] generalized the notion of chain recurrence on the theory of dynamical systems without uniqueness. Hurley $[9,10,11]$ introduced the notion of chain recurrence on noncompact spaces and obtained some results about the chain recurrence on arbitrary metric spaces. Also, he introduced the alternative definition of chain recurrence for semiflow and proved that the alternative definition is equivalent to the usual definition. Recently, Chu and Park extended the concept of chain recurrence to the multi-valued dynamical systems [5, 6]. In [12], Kościelniak showed that the chain recurrent sets are Cantor sets in the space of $\mathbb{Z}^{2}$-actions on the unit interval. Oprocha [14] extended the chain recurrent set for the space of $\mathbb{Z}^{d}$-actions.

In [13], Newhouse proved that any residual subset of the set of $C^{r}$-diffeomorphisms $(r>1)$ on $X$ must contain diffeomorphisms $f$ with the number of chain components of $f$ is infinite. Conley [4] described chain recurrent set in terms of attractors and showed that the chain recurrent mapping is upper

Received August 18, 2008.

2000 Mathematics Subject Classification. 37B20, 54H20.

Key words and phrases. chain recurrence, residual set, flow.

(C)2010 The Korean Mathematical Society 
semicontinuous. Moreover, Hurley [7] obtained the residual subset $J$ of the space of $C^{1}$-diffeomorphisms on any compact Riemannian manifold $X$ such that the maps

$$
\begin{aligned}
& f \mapsto \text { chain recurrent set for } f, \\
& f \mapsto \text { number of chain components for } f
\end{aligned}
$$

are continuous on $J$.

The purpose of this paper is to extend the above result to continuous flows on a compact metric space $X$.

Throughout this paper, $X$ denote a compact metric space with metric $d$.

\section{Definitions and lemmas}

A flow on $X$ is a continuous map $\phi: X \times(-\infty, \infty) \rightarrow X$ satisfying (1) $\phi(x, 0)=x$ for all $x \in X$ and $(2) \phi(\phi(x, s), t)=\phi(x, s+t)$ for all $x \in X$ and for all $s, t \in \mathbb{R}$. We denote $\Phi(X)$ the set of all continuous flows on $X$. Let $\varepsilon>0$ and $t>0$. For any $x, y \in X$, we say that a sequence $\left\{\left(x_{i}, t_{i}\right)\right\}_{i=1}^{n}$ is an $(\varepsilon, t)$-chain from $x$ to $y$ for $\phi \in \Phi(X)$ if

(1) $x_{1}=x$ and $t_{i} \geq t$ for all $i=1, \ldots, n$

(2) $d\left(\phi\left(x_{i}, t_{i}\right), x_{i+1}\right)<\varepsilon$ for all $i=1, \ldots, n-1$ and $d\left(\phi\left(x_{n}, t_{n}\right), y\right)<\varepsilon$.

In case of $t=1$, that is $(\varepsilon, 1)$-chain, we say that it is an $\varepsilon$-chain. So we can consider a canonical equivalence relation on $X$. Two points $x$ and $y$ are said to be chain equivalent if for every $\varepsilon>0$ and every $t>0$, there exists an $(\varepsilon, t)$ chain from $x$ to $y$ and there exists an $(\varepsilon, t)$-chain from $y$ to $x$. A point $x$ in $X$ is called chain recurrent with respect to $\phi$ if $x$ is chain equivalent to itself. The set of chain recurrent points of $\phi \in \Phi(X)$, denoted by $C R(\phi)$, is called the chain recurrent set of $\phi$. It is clear that the chain equivalence relation is an equivalence relation on $C R(\phi)$. It is possible that the notion of chain equivalence is changed to more simple definition, in detail, two points $x, y$ in $C R(\phi)$ are chain equivalent if and only if for any $\varepsilon>0$ there exist two $\varepsilon$-chains from $x$ to $y$ and from $y$ to $x$. An equivalence class under this equivalence relation for $\phi$ is called a chain component of $\phi$. It turns out for flows on compact manifolds that the chain components are exactly the connected components of $C R(\phi)$. See [8].

To prove our theorem, we need some definitions and lemmas. Firstly, we define a function $\rho: \Phi(X) \times \Phi(X) \rightarrow \mathbb{R}$ by setting

$$
\rho(\phi, \psi)=\sup \left\{\min \left\{\max \{d(\phi(x, t), \psi(x, t)): x \in X,-T \leq t \leq T\}, \frac{1}{T}\right\}: T>0\right\}
$$

for all $\phi, \psi \in \Phi(X)$.

Lemma 2.1. $\rho$ is a metric on $\Phi(X)$.

Proof. Put $M_{T}(\phi, \psi)=\max \{d(\phi(x, t), \psi(x, t)): x \in X,-T \leq t \leq T\}$. 
Now, let $\varepsilon>0$. Since $\rho(\phi, \psi)-\varepsilon<\rho(\phi, \psi)$, we have

$$
\min \left\{M_{T}(\phi, \psi) \frac{1}{T}\right\}>\rho(\phi, \psi)-\varepsilon \quad \text { for some } T>0 .
$$

Then there are $y \in X$ and $s \in[-T, T]$ such that $d(\phi(y, s), \psi(y, s))=M_{T}(\phi, \psi)$. Let us check the triangle inequality $\rho(\phi, \gamma)+\rho(\gamma, \psi) \geq \rho(\phi, \psi)$. If $M_{T}(\phi, \gamma) \geq$ $\frac{1}{T}$, then we have

$$
\begin{aligned}
\rho(\phi, \gamma)+\rho(\gamma, \psi) & \geq \rho(\phi, \gamma) \\
& \geq \min \left\{M_{T}(\phi, \gamma), \frac{1}{T}\right\}=\frac{1}{T} \\
& \geq \min \left\{M_{T}(\phi, \psi), \frac{1}{T}\right\} \\
& >\rho(\phi, \psi)-\varepsilon .
\end{aligned}
$$

If $M_{T}(\gamma, \psi) \geq \frac{1}{T}$, then we have

$$
\begin{aligned}
\rho(\phi, \gamma)+\rho(\gamma, \psi) & \geq \rho(\gamma, \psi) \\
& \geq \min \left\{M_{T}(\gamma, \psi), \frac{1}{T}\right\}=\frac{1}{T} \\
& \geq \min \left\{M_{T}(\phi, \psi), \frac{1}{T}\right\} \\
& >\rho(\phi, \psi)-\varepsilon .
\end{aligned}
$$

On the other hand, if $M_{T}(\phi, \gamma)<\frac{1}{T}$ and $M_{T}(\gamma, \psi)<\frac{1}{T}$, then we have

$$
\begin{aligned}
\rho(\phi, \gamma)+\rho(\gamma, \psi) & \geq \min \left\{M_{T}(\phi, \gamma), \frac{1}{T}\right\}+\min \left\{M_{T}(\gamma, \psi), \frac{1}{T}\right\} \\
& =M_{T}(\phi, \gamma)+M_{T}(\gamma, \psi) \\
& \geq d(\phi(y, s), \gamma(y, s))+d(\gamma(y, s), \psi(y, s)) \\
& \geq d(\phi(y, s), \psi(y, s))=M_{T}(\phi, \psi) \\
& \geq \min \left\{M_{T}(\phi, \psi), \frac{1}{T}\right\} \\
& >\rho(\phi, \psi)-\varepsilon .
\end{aligned}
$$

Since $\varepsilon$ is arbitrary, we have $\rho(\phi, \gamma)+\rho(\gamma, \psi) \geq \rho(\phi, \psi)$. Other conditions for $\rho$ can be easily verified. The proof is completed.

Define $C^{0}(X)$ by the set of all continuous flows of $X$ to itself with the metric $\rho$.

Lemma 2.2 (Integral continuity theorem). Let $\phi$ be a flow on metric space $X$. Let $x \in X$ and $K$ be a compact subset of $\mathbb{R}^{+}$. For every positive number $\varepsilon$, there is a positive number $\delta$ such that $d(x, y)<\delta$ implies $d(\phi(x, t), \phi(y, t))<\varepsilon$, for every $t \in K$.

Proof. The proof is similar to the proof of original integral continuity theorem. 
Let $X_{1}$ be a metric space and $X_{2}$ a compact metric space. And let $F\left(X_{2}\right)$ be the set of all closed nonempty subsets of $X_{2}$ with the Hausdorff metric

$$
d_{H}(A, B)=\max \left\{\sup _{a \in A} d(a, B), \sup _{b \in B} d(A, b)\right\}, A, B \in F\left(X_{2}\right) .
$$

A map $f: X_{1} \rightarrow F\left(X_{2}\right)$ is called upper(lower) semicontinuous at $x \in X_{1}$ if for any $\varepsilon>0$ there exists $\delta>0$ such that if $d(x, y)<\delta$, then $f(y) \subset$ $B(f(x), \varepsilon)(f(x) \subset B(f(y), \varepsilon))$ respectively. Recall that a subset $S$ of a topological space $X$ is residual if $S$ can be realized as a countable intersection of open dense subsets of $X$.

Remark 2.3 ([15]). A map $f: X_{1} \rightarrow F\left(X_{2}\right)$ is lower semicontinuous at $x \in X_{1}$ if and only if for any open subset $U$ of $X_{2}$ with $U \cap f(x) \neq \emptyset$, there exists a neighborhood $V$ of $x$ in $X_{1}$ such that $U \cap f(y) \neq \emptyset$ for all $y \in V$.

Similarly, $f$ is upper semicontinuous at $x \in X_{1}$ if and only if for any open neighborhood $U$ of $f(x)$, there exists a neighborhood $V$ of $x$ in $X_{1}$ such that $f(y) \subseteq U$ for all $y \in V$.

Lemma $2.4([7])$. Let $f: X_{1} \rightarrow F\left(X_{2}\right)$ be either upper or lower semicontinuous. Then the set of all continuity points of $f$ is a residual subset of $X_{1}$.

Lemma 2.5. Assume that a sequence $\left(\phi_{n}\right)$ converges to $\phi$ in $C^{0}(X)$ a sequence $\left(x_{n}\right)$ converges to $x$ and a sequence $\left(y_{n}\right)$ also converges to $y$ in $X$. Suppose that every positive integer $n$ and positive number $\varepsilon$, there exists an $\varepsilon$-chain for $\phi_{n}$ from $x_{n}$ to $y_{n}$. Then for every $\varepsilon>0$, there is an $\varepsilon$-chain for $\phi$ from $x$ to $y$.

Proof. Let $0<\varepsilon<1$ be arbitrary. For any $a \in X$, by integral continuity theorem, there exists $0<\delta(a)<\frac{\varepsilon}{3}$ such that if $d(a, x)<\delta(a)$, then $d(\phi(a, t), \phi(x, t))<\frac{\varepsilon}{12}$ for all $1 \leq t \leq 2$. Then $\left\{B\left(a, \frac{\delta(a)}{2}\right): a \in X\right\}$ is an open cover of $X$. Since $X$ is compact, there are finitely many points $a_{1}, \ldots, a_{n} \in X$ such that $X=\bigcup_{i=1}^{n} B\left(a_{i}, \frac{\delta\left(a_{i}\right)}{2}\right)$. Put $\delta=\frac{1}{2} \min \left\{\delta\left(a_{1}\right), \ldots, \delta\left(a_{n}\right)\right\}$ and let $d(x, y)<\delta$. Then there exists $i$ such that $x \in B\left(a_{i}, \frac{\delta\left(a_{i}\right)}{2}\right)$. Since $d\left(a_{i}, x\right)<$ $\frac{\delta\left(a_{i}\right)}{2}, d\left(\phi\left(a_{i}, t\right), \phi(x, t)\right)<\frac{\varepsilon}{12}$ for all $1 \leq t \leq 2$. Since

$$
d\left(a_{i}, y\right) \leq d\left(a_{i}, x\right)+d(x, y)<\frac{\delta\left(a_{i}\right)}{2}+\delta \leq \frac{\delta\left(a_{i}\right)}{2}+\frac{\delta\left(a_{i}\right)}{2}=\delta\left(a_{i}\right),
$$

we have $d\left(\phi\left(a_{i}, t\right), \phi(y, t)\right)<\frac{\varepsilon}{12}$ for all $1 \leq t \leq 2$. Thus we obtain that

$$
d(\phi(x, t), \phi(y, t)) \leq d\left(\phi(x, t), \phi\left(a_{i}, t\right)\right)+d\left(\phi\left(a_{i}, t\right), \phi(y, t)\right)<\frac{\varepsilon}{12}+\frac{\varepsilon}{12}=\frac{\varepsilon}{6}
$$

for all $1 \leq t \leq 2$.

Let $\rho(\phi, \psi)<\frac{\varepsilon}{6}$. Since $\frac{1}{6}>\frac{\varepsilon}{6}>\rho(\phi, \psi) \geq \min \left\{M_{6}(\phi, \psi), \frac{1}{6}\right\}$, we have $M_{6}(\phi, \psi)<\frac{\varepsilon}{6}$. Thus $d(\phi(x, t), \psi(x, t))<\frac{\varepsilon}{6}$ for all $x \in X$ and all $-6 \leq t \leq 6$. Hence if $d(x, y)<\delta$, then we have

$$
d(\phi(x, t), \psi(y, t)) \leq d(\phi(x, t), \phi(y, t))+d(\phi(y, t), \psi(y, t))<\frac{\varepsilon}{6}+\frac{\varepsilon}{6}=\frac{\varepsilon}{3}
$$


for all $1 \leq t \leq 2$. Choose a positive integer $m$ such that $\rho\left(\phi, \phi_{m}\right)<\frac{\varepsilon}{3}, d\left(x, x_{m}\right)$ $<\delta$ and $d\left(y, y_{m}\right)<\delta$. By assumption, we select $\frac{\varepsilon}{3}$-chain $\left\{\left(z_{i}, t_{i}\right)\right\}_{i=1}^{n}$ for $\phi_{m}$ from $x_{m}$ to $y_{m}$. We may assume that $1 \leq t_{i} \leq 2$ for all $i$. Since $d\left(x, x_{m}\right)<\delta$, we have $d\left(\phi\left(x, t_{1}\right), \phi_{m}\left(x_{m}, t_{1}\right)\right)<\frac{\varepsilon}{3}$. Since $\bar{d}\left(\phi_{m}\left(x_{m}, t_{1}\right), z_{2}\right)=d\left(\phi_{m}\left(z_{1}, t_{1}\right), z_{2}\right)<\frac{\varepsilon}{3}$, we get

$$
d\left(\phi\left(x, t_{1}\right), z_{2}\right) \leq d\left(\phi\left(x, t_{1}\right), \phi_{m}\left(x_{m}, t_{1}\right)\right)+d\left(\phi_{m}\left(x_{m}, t_{1}\right), z_{2}\right)<\frac{\varepsilon}{3}+\frac{\varepsilon}{3}<\varepsilon .
$$

Since $d\left(\phi_{m}\left(z_{i}, t_{i}\right), z_{i+1}\right)<\frac{\varepsilon}{3}$ and $d\left(\phi_{m}\left(z_{i}, t_{i}\right), \phi\left(z_{i}, t_{i}\right)\right)<\frac{\varepsilon}{3}$ for all $1 \leq i \leq n-1$, we have $d\left(\phi\left(z_{i}, t_{i}\right), z_{i+1}\right)<\varepsilon$ for all $1 \leq i \leq n-1$. Then we get

$$
\begin{aligned}
d\left(\phi\left(z_{n}, t_{n}\right), y\right) & \leq d\left(\phi\left(z_{n}, t_{n}\right), \phi_{m}\left(z_{n}, t_{n}\right)\right)+d\left(\phi_{m}\left(z_{n}, t_{n}\right), y_{m}\right)+d\left(y, y_{m}\right) \\
& <\frac{\varepsilon}{3}+\frac{\varepsilon}{3}+\delta<\varepsilon
\end{aligned}
$$

Thus $\left\{\left(x, t_{1}\right),\left(z_{2}, t_{2}\right), \ldots,\left(z_{n}, t_{n}\right)\right\}$ is an $\varepsilon$-chain for $\phi$ from $x$ to $y$. This completes the proof.

\section{Main results}

Now, we consider a map $C R$ sending a continuous flow $\phi$ in $C^{0}(X)$ to a chain recurrent set $C R(\phi)$ for $\phi$. Also we consider a function $N$ from a continuous flow $\phi$ in $C^{0}(X)$ to a number $N(\phi)$ of chain components in the extended half line $[0, \infty]$ (viewed as the one-point compactification of $[0, \infty)$ ). The following is our main theorem.

Main Theorem. There is a residual subset $R$ of $C^{0}(X)$ such that the maps $C R$ and $N$ are continuous at each point of $R$.

Proof. Firstly, we will show that there is a residual subset $R_{1}$ of $C^{0}(X)$ such that the map $C R$ is continuous at each point of $R_{1}$. Suppose that $C R$ is not upper semicontinuous at $\phi \in C^{0}(X)$. Then we can choose $\varepsilon>0$ such that for each $n>0$ there exists $\phi_{n} \in C^{0}(X)$ such that $\rho\left(\phi_{n}, \phi\right)<\frac{1}{n}$ and $C R\left(\phi_{n}\right) \nsubseteq$ $B(C R(\phi), \varepsilon)$. Thus we can select $x_{n} \in C R\left(\phi_{n}\right)$ satisfying $d\left(x_{n}, C R(\phi)\right) \geq \varepsilon$. We may assume that $x_{n} \rightarrow x \in X$. Then we have $d(x, C R(\phi)) \geq \varepsilon$. Since $\phi_{n} \rightarrow \phi$ and $x_{n} \in C R\left(\phi_{n}\right)$, by Lemma 2.5, we get $x \in C R(\phi)$. This is a contradiction, which completes the assertion. From Lemma 2.4, there is a residual subset $R_{1}$ of $C^{0}(X)$ such that $C R$ is continuous at each point of $R_{1}$.

Next, we are going to show that there is a residual subset $R$ of $R_{1}$ such that the map $N$ is continuous at each point of $R$. It is enough to show that the map $N: R_{1} \rightarrow[0, \infty]$ is lower semicontinuous. Let $\phi \in R_{1}$. There are two cases depending upon whether $N(\phi)<\infty$ or not. In the first case $N(\phi)$ is finite. Thus we can list the $\phi$-chain components $M_{1}, \ldots, M_{k}$. Then there is $\varepsilon>0$ such that $B\left(M_{i}, \varepsilon\right) \cap B\left(M_{j}, \varepsilon\right)=\emptyset$ if $i \neq j$. Since the map $C R$ is continuous, by Remark 2.3, we can choose $\delta>0$ such that if $\rho(\phi, \psi)<\delta$, then $C R(\psi) \subset B(C R(\phi), \varepsilon)$ and $C R(\psi) \cap B\left(M_{i}, \varepsilon\right) \neq \emptyset$ for all $i=1, \ldots, k$. Let $A$ be 
a chain component of $C R(\psi)$. Then we have

$$
A \subset C R(\psi) \subset B(C R(\phi), \varepsilon) \subset \bigcup_{i=1}^{k} B\left(M_{i}, \varepsilon\right) .
$$

Thus we obtain

$$
A=A \cap \bigcup_{i=1}^{k} B\left(M_{i}, \varepsilon\right)=\bigcup_{i=1}^{k}\left(A \cap B\left(M_{i}, \varepsilon\right)\right) .
$$

Since $A$ is a connected component in $C R(\psi)$, there exists a unique $i$ satisfying $A \cap B\left(M_{i}, \varepsilon\right) \neq \emptyset$. Hence we have $A \subset B\left(M_{i}, \varepsilon\right)$. For any $i=1, \ldots, k$, since $C R(\psi) \cap B\left(M_{i}, \varepsilon\right) \neq \emptyset$, we can choose at least one chain component $B$ of $C R(\psi)$ such that $B \subset B\left(M_{i}, \varepsilon\right)$. This implies that $N(\phi) \leq N(\psi)$.

In the remaining case $N(\phi)$ is infinite. For each $n \in \mathbb{Z}^{+}$, we can choose $\delta(n)>0$ such that if $\rho(\phi, \psi)<\delta$, then $N(\psi) \geq n$. This means that $\lim _{\psi \rightarrow \phi} N(\psi)$ is also infinite. Hence $N$ is lower semicontinuous at $\phi$. By Lemma 2.4, we can find the residual subset $R$ of $R_{1}$ satisfying $C R$ and $N$ are continuous at each point of $R$. Since a residual subset of residual set is also residual, the proof is completed.

\section{References}

[1] L. Block and John E. Franke, The chain recurrent set for maps of the interval, Proc. Amer. Math. Soc. 87 (1983), no. 4, 723-727.

[2] I. U. Bronstein and A. Ya. Kopanskii, Chain recurrence in dynamical systems without uniqueness, Nonlinear Anal. 12 (1988), no. 2, 147-154.

[3] C. Conley, The gradient structure of a flow, I. B. M. Res. RC 3932. Yorktown Heights, N.Y., 1972.

[4] _ Isolated Invariant Sets and the Morse Index, CBMS Regional Conference Series in Mathematics, 38. American Mathematical Society, Providence, R.I., 1978.

[5] H. Chu, Chain recurrence for multi-valued dynamical systems on noncompact spaces, Nonlinear Anal. 61 (2005), no. 5, 715-723.

[6] H. Chu and J. Park, Attractors for relations in $\sigma$-compact spaces, Topology Appl. 148 (2005), no. 1-3, 201-212.

[7] M. Hurley, Bifurcation and chain recurrence, Ergodic Theory Dynam. Systems 3 (1983), no. $2,231-240$

[8] _ Fixed points of topologically stable flows, Trans. Amer. Math. Soc. 294 (1986), no. $2,625-633$

[9] Chain recurrence and attraction in noncompact spaces, Ergodic Theory Dynam. Systems 11 (1991), no. 4, 709-729.

[10] , Noncompact chain recurrence and attraction, Proc. Amer. Math. Soc. 115 (1992), no. 4, 1139-1148.

[11] Chain recurrence, semiflows, and gradients, J. Dynam. Differential Equations 7 (1995), no. 3, 437-456.

[12] P. Kościelniak, Generic properties of $\mathbb{Z}^{2}$-actions on the interval, Topology Appl. 154 (2007), no. 14, 2672-2677.

[13] S. Newhouse, Diffeomorphisms with infinitely many sinks, Topology 13 (1974), 9-18.

[14] P. Oprocha, Chain recurrence in multidimensional time discrete dynamical systems, Discrete Contin. Dyn. Syst. 20 (2008), no. 4, 1039-1056. 
[15] J. Park, D. Kang, and H. Chu, Stabilities in multi-valued dynamical systems, Nonlinear Anal. 67 (2007), no. 7, 2050-2059.

Jong-SuH PARK

Department of Mathematics

Chungnam National University

DAEJEON 305-764, KoREA

E-mail address: jspark@math.cnu.ac.kr

SE-Hyun Ku

Department of Mathematics

Chungnam National University

DAEJEON 305-764, Korea

E-mail address: shku@cnu.ac.kr 